\title{
Albendazole regulates radiosensitivity of human pancreatic cancer cells by inhibiting HIF-1 $a$ and basic fibroblast growth factor
}

\section{Haifeng Chen}

First Affiliated Hospital of Soochow University https://orcid.org/0000-0002-1088-4976

\section{Xiaochong Zhou}

First Affiliated Hospital of Soochow University

\section{Zhen Weng}

Soochow University

\section{Xing Wei}

First Affiliated Hospital of Soochow University

Chunfang Xu ( $\nabla$ chf6607@163.com)

\section{Yang He}

Soochow University

\section{Research}

Keywords: Pancreatic cancer, Albendazole, HIF-1a, bFGF, Radiosensitivity

Posted Date: January 6th, 2020

DOI: https://doi.org/10.21203/rs.2.20141/v1

License: (9) This work is licensed under a Creative Commons Attribution 4.0 International License. Read Full License 


\section{Abstract}

Background Albendazole, a clinical antiparasitic drug, has been shown to have antitumor activity and supress expression of hypoxia-inducible factor 1-alpha. While hypoxia, the most prominent feature of tumor microenvironment, is associated with radiotherapy tolerance. Herein, we aimed to identify Albendazole as a candidates that improves tumor microenvironment and enhances the radiosensitivity of human pancreatic cancer cells.

Methods MTT assay, clone formation and flow cytometry were performed to assess the effect of ABZ and radiation on $\mathrm{PC}$ cell line proliferation and apoptosis induction. In addition, the expression levels of hypoxia-inducible factor 1-alpha (HIF-1a) and basic fibroblast growth factor (bFGF) were assessed using western blotting. Finally, the effects of $A B Z$ on tumor growth and radiosensitivity were examined using nude mice xenograft model.

Results ABZ significantly improved hypoxia-induced radiation resistance in PC cell line PATU8988 and SW1990 as evidenced by decreased absorbance of MTT, reduced colony number, and increased apoptotic cell ratio. Furthermore, the in vivo results confirmed that $A B Z$ suppressed tumor growth. On mechanisms, treatment with ABZ decreased HIF-1 $a$ and bFGF expression levels, which correlated with radioresistance in cells exposed to hypoxia in vitro and tumor to radiation in vivo.

Conclusion Taken together, our datas show that HIF-1a and bFGF regulate radiation sensitivity in PC cells under hypoxic conditions. And ABZ enhances radiosensitivity of pancreatic cancer by suppression of HIF$1 \mathrm{a}$ and bFGF expression.

\section{Background}

Pancreatic cancer is a highly malignant tumor with high invasiveness [1, 2]. Due to late diagnosis and poor prognosis, most PC patients are fit for surgical resection at the time of diagnosis. This leads to high mortality rate in PC, making it the fourth leading cause of cancer-related deaths. Of note, the incidence of PC has been on the rise globally [3] [4] [5]. Radiotherapy, chemotherapy and surgical resection are the main treatment approaches for pancreatic cancer patients [3]. Radiation therapy plays a key role in treatment of unresectable locally advanced and intolerable surgical resection pancreatic cancer patients [6]. The emergence of stereotactic body radiotherapy has accelerated the application of radiotherapy in cancer treatment [7].

However, tumor resistance caused by the tumor microenvironment compromises the efficacy of radiotherapy. Hypoxia is the most prominent feature in many solid tumors and is associated with poor prognosis and clinical resistance to radiotherapy in pancreatic cancer patients [8]. Elevated expression of HIF-1a was found in tumor tissue under hypoxic microenvironment and is associated with tumor radiotherapy resistance, development and invasion $[9,10]$. In addition, hypoxia up-regulates bFGF expression, which enhances the cancer cell growth and increases the radiation resistance of cancer cells $[11,12]$. Further, bFGF promotes the expression of hypoxia-inducible factor $1 \mathrm{a}$ by modifying the tumor 
matrix environment, an effect that aggravates the tumor hypoxia [13]. Therefore, we explored the effects of $A B Z$ on the expression of HIF-1 $a$ and bFGF in tumors, and tumor radiotherapy resistance.

ABZ a benzimidazole carbamate (methyl 5-propylthio-1H-benzimidazole-2-yl carbamate) is clinically used to treat human and animal helminth parasites infections[14]. The antitumor effect of $A B Z$ on various cancers has been reported in several tumors $[15,16]$. However, the effect of $A B Z$ on the tumor microenvironment has not been studied in depth, especially tumor fiber microenvironment. In this study, we investigated the effects of ABZ on HIF-1 $a$ and bFGF expression levels, the key factors for tumor microenvironment, and explored the link between the two factors.

\section{Materials And Methods}

\section{Materials and antibodies}

ABZ, dimethyl sulfoxide (DMSO), penicillin, cobalt chloride ( $\mathrm{CoCl} 2)$ and triton-100 were purchased from Sigma Aldrich chemical Co., Ltd (St. Luis, MO, USA). Annexin V-FITC apoptosis detection kit, 3- (4,5dimethylthiazol-2-yl)-2,5 diphenyl tetrazolipM bromide (MTT), Colorimetric TUNEL Apoptosis Assay kit, Hematoxylin and Eosin Staining Kit and RIPA lysis buffer were purchased from Beyotime Institute of Biotechnology, Nantong, Jiangsu, China. Cell culture materials such as Dulbecco's modification of Eagle's medipM (DMEM) and Fetal Bovine Serum (FBS) were obtained from Gibco BRL, CA, USA. The nuclear antigen Ki67 was purchased from Cell Signaling Technology (CST). The Sirius Red staining kit was obtained from Yuanye Bio-Technology. All reagents were of $100 \%$ purity or analytical grade.

\section{Cell culture}

The human pancreatic cancer cell lines, PATU8988 and SW1990 were obtained from American type cell

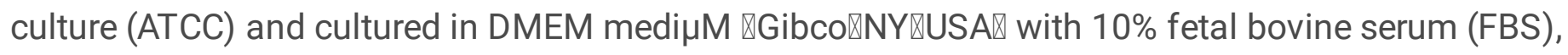
penicillin $(100 \mathrm{U} / \mathrm{ml})$, and streptomycin $(100 \mu \mathrm{g} / \mathrm{ml})(\mathrm{P} / \mathrm{S})$ and incubated at $37^{\circ} \mathrm{C}$ and $5 \% \mathrm{CO}_{2}$. Cobalt Chloride $\left(\mathrm{CoCl}_{2}, 50 \mu \mathrm{M}\right)$ was used to induce chemical hypoxia environment.

\section{Western bolt analysis}

PATU8988 and SW1990 proteins were used for western blot analysis as described

previously [17]. Equal amounts of protein were separated using SDS-PAGE and then transferred onto PVDF membrane. The protein was blocked with $5 \%$ skim milk and incubated with the primary antibody (HIF-1a, 1:2000; Abcam, Cambridge, UK and FGF-2, 1:1000; Abcam, Cambridge, UK) in PBST for 2h at room temperature. The membrane was incubated with secondary antibody (Goat Anti-Rabbit IgG HRP, Bioworld Technology, Lot NO: AB21171, Dilution:1:10000;) in PBST for $1 \mathrm{hrs.} \mathrm{GAPDH} \mathrm{was} \mathrm{used} \mathrm{as} \mathrm{an}$ internal control. Proteins were visualized by ECL kit (Beyotime).

\section{MTT analysis}


Cells were seeded on 96 -well plates at the concentration of $8 \times 10^{3}$ cells/well. After 24 hours, the cells were treated with serial concentrations of $\mathrm{ABZ}$ or $\mathrm{CoCl}_{2}$ for 24 and 48 hours, followed by incubation with $20 \mu \mathrm{l}$ MTT solution $(0.5 \mathrm{mg} / \mathrm{mL})$ for $4 \mathrm{hrs}$ at $37^{\circ} \mathrm{C}$, washed in solution, and dissolved in $200 \mu \mathrm{L}$ DMSO. The absorbance value was measured at a wavelength of $490 \mathrm{~nm}$.

\section{Colony formation analysis}

Pancreatic cancer cells (200 cells per well) were seeded into six-well plates. After 24 hours, the cells were exposed to normoxia and Cobalt Chloride $\left(\mathrm{CoCl}_{2}, 50 \mu \mathrm{M}\right)$ to induce hypoxia in the presence or absence of ABZ (200 nM) for $24 \mathrm{hrs}$. Cells were then treated with 4 Gy of X-ray irradiation.

After incubation for 20 days, the colonies were fixed and stained with Giemsa dye for $15 \mathrm{~min}$, followed by 3 times' PBS wash and colonies (>50 cells/colony) counting under a microscopy.

\section{X-ray irradiation}

Cell irradiation was performed using X-RAD320iX (Precision X-Ray, North Branford, CT, USA) at a dose of 4Gy with $8.15-\mathrm{mA}$ X-ray, $1.5 \mathrm{~Gy} / \mathrm{min}$. Prior to irradiation, the cells were treated in normoxia and Cobalt Chloride $\left(\mathrm{CoCl}_{2}, 50 \mu \mathrm{M}\right)$ to induce hypoxia in the presence or absence of $A B Z(200 \mathrm{nM})$. Before each experiment, the mice were anesthetized by intraperitoneal injection of chloral hydrate, fixed on a special plate, exposed at the right front leg with the other body parts covered with a lead plate, and the mouse was placed in an irradiation apparatus.

\section{Flow cytometric analysis of apoptosis}

To detect apoptosis effects, Annexin V-FITC was used to stain PATU8988 and SW1990 cells treated in normoxia and $\mathrm{CoCl}_{2}(50 \mu \mathrm{M})$-induced hypoxia conditions with $\mathrm{ABZ}(200 \mathrm{nM})$ or control for $24 \mathrm{hrs}$. Cells were, then, treated with 4Gy of X-ray irradiation. After 24 hours of incubation, cells were harvested and resuspended with $100 \mu \mathrm{L} 1 \mathrm{x}$ binding buffer, followed by Annexin V-FITC and PI Staining before subjection to flow cytometry analysis.

\section{Xenograft nude mice model}

Six-week-old male BALB/c nude mice were obtained from Beijing Vitallihua. This study protocol was approved by the Institutional Animal Care and Usage Committee of Soochow University. PATU8988 cell line $\left(5 \times 10^{6}\right)$ were subcutaneously inoculated into the right flank of nude mice. When tumors size reached 5-10 mm in diameter, the mice were randomly divided into four different groups $(n=6)$ : Vehicle group (sesame oil), ABZ treatment group, Irradiation treatment group and ABZ and irradiation co-treatment group. As described by TC Hardin [18], the second and fourth groups received $300 \mathrm{mg} / \mathrm{kg}$ twice daily oral gavage $A B Z$ suspended in sesame oil, whereas the control group was treated with the vehicle control (sesame oil) for 20 days. Tumor irradiation was performed using X-RAD320iX (Precision X-Ray, North Branford, CT, USA) at a dose of 4Gy with 8.15-mA X-ray, 1.5Gy/min on days 8, 15, 22. The tumor volumes 
were measured every day with a vernier calipers on day 8 and were calculated using the formula: tumor volume $=a b^{2} / 2$, where "a" was the longest tumor diameter and "b" was the shortest tumor diameter measured. The PATU8988 derived tumors were harvested on the $27^{\text {th }}$ day from the mice and fixed in $4 \%$ formaldehyde solution for pathological analyses or lysed in RIPA lysis buffer for western bolt analyses.

\section{Immunohistochemical analysis}

The fixed tumors were placed in paraffin blocks. Paraffin sections were examined for the expression of ki67 and HIF-1a antigen by immunohistochemistry. As described by Krajewski S [19], tumor tissue paraffin section were deparaffinized in xylene, hydrated in gradient alcohol, followed by antigen retrieval in boiling $0.1 \%$ citrate buffer $(\mathrm{pH} 6.0)$ for $30 \mathrm{~min}$ and endogenous peroxidase removal by $3 \% \mathrm{H}_{2} \mathrm{O}_{2}$. Further, sections were washed three times in phosphate-buffered saline, blocked with $10 \%$ fetal bovine serum (Bisharp) containing 1\% Tween 20 (TBST) at room temperature for $1 \mathrm{hr}$ and exposed to mice antiki67 $(1 / 100)$ primary antibody overnight at $4{ }^{\circ} \mathrm{C}$. Finally, the expression of the antigen was visualized by HRP conjugated secondary antibody and DAB substrate exposure.

\section{TUNEL Apoptosis Assay}

Colorimetric TUNEL Apoptosis Assay kit was used to measure tumor apoptosis. Tumor tissue paraffin sections were deparaffinized in xylene, hydrated in gradient alcohol, followed by permeabilization of cell membrane with $20 \mu \mathrm{g} / \mathrm{ml}$ proteinase $\mathrm{K}$ for $30 \mathrm{~min}$ and endogenous peroxidase removal by $3 \% \mathrm{H}_{2} \mathrm{O}_{2}$. The sections were rinsed three times with PBS, incubated with a mix solution containing the enzyme terminal deoxynucleotide transferase (TdT) and biotinylated (Bio-16) dUTP in TdT buffer in a humid atmosphere at $37^{\circ} \mathrm{C}$ for $60 \mathrm{~min}$ and the reaction was terminated with stop buffer for $10 \mathrm{~min}$ at $37^{\circ} \mathrm{C}$. After rinsing with PBS, the slides were incubated with streptavidin-HRP conjugate (30 min, RT), stained with diaminobenzidine for $10 \mathrm{~min}$ and counterstained with hematoxylin.

\section{Sirius red assay}

Sirius red assays were performed as described by Grimm PC [20]. Pancreatic xenograft tumor tissues fixed in $4 \%$ formaldehyde were embedded in paraffin for pathological analysis, and then stained with the Sirius Red Kit (Yuanye BioTechnology, Shanghai, China) for $1 \mathrm{hr}$. Subsequently, the sections were washed with water for $5 \mathrm{~min}$, counterstained with Mayer's hematoxylin, and visualization done under an inverted microscope.

\section{Statistical analysis}

All statistical analyses were performed with GraphPad Prism software (GraphPad Software, Inc, La Jolla, CA). All results were presented as mean \pm standard deviation (SD). Student t-test and one-way ANOVA assay was used for between group comparison and multiple group comparisons. All $P$ values $<0.05$ was considered statistically significant. 


\section{Results}

\section{Radiation dosage, ABZ concentration and hypoxia condition optimization}

In order to observe evaluate the effect of $A B Z$ against radiation resistance of PC cells exposed to hypoxia, PATU8988 and SW1990 human pancreatic cancer cell lines were firstly treated with series dosage of radiation and series concentration of $A B Z$. As shown in Fig.1A\&B, a significant inhibition on cell viability could be achieved at $4 \mathrm{~Gy}$ dosage in both cell lines at $48 \mathrm{~h}$ time points. Moreover, we also found that at the concentration of $200 \mathrm{nM} \mathrm{ABZ}$, a significant inhibition on the PC cell viability could be achieved (Fig. $1 C \& D)$. In addition, cobalt chloride $\left(\mathrm{CoCl}_{2}\right)$ was employed to mimic hypoxia condition, and we observed a significant induction of the HIF-1a without affecting the cell viability at the concentration of $50 \mu \mathrm{M}$ (Fig.2A, B, C\&D). Therefore, $4 \mathrm{~Gy}$ radiation and $200 \mathrm{nM} \mathrm{ABZ}$ were used as the treatment modality, whereas $50 \mu \mathrm{M} \mathrm{CoCl}_{2}$ was employed as the hypoxia induction condition in the following experiment setting.

\section{ABZ improves radiation resistance of hypoxia pancreatic cancer cells lines}

The colony formation rate for cells exposed to normoxia condition significantly decreased in comparison to cells cultured in chemical hypoxia environment after X-ray radiation, indicating their resistance to radiation. Interestingly, $A B Z$ improved the radiosensitivity of human pancreatic cancer cells under hypoxia condition (Fig.2A and B). Moreover, we explored the effects of ABZ on apoptosis of PC cells exposed to normoxia and hypoxia conditions in the presence of $\mathrm{X}$-ray radiation. We observed that cell apoptosis rate was lower under hypoxia condition than under normoxia condition and ABZ significantly enhanced the apoptosis rate both in normoxia and hypoxia condition (Fig.2C). These results indicated that besides the killing effects by $A B Z$ at normoxia condition, $A B Z$ could also improve radiation resistance of hypoxia pancreatic cancer cells lines.

\section{ABZ promotes the radiosensitivity of PATU8988 xenograft growth in vivo}

To explore the potential radiosensitization effect of $A B Z$ on human pancreatic cancer cells in vivo, we subcutaneously injected PATU8988 cells into the right armpit of BALB/c nude mice to establish a xenograft model. The mice were, then, treated with the vehicle (sesame oil), ABZ at $300 \mathrm{mg} / \mathrm{kg}(\mathrm{n}=6)$ by oral gavage every day or X-ray at a dose of 4Gy on day 8 and 15., After treatment for 21 days, mice were sacrificed, and tumor tissues harvested and photographed (Fig.3A). ABZ and irradiation co-treatment group was more effective in suppressing tumor growth compared to control group, ABZ-treated group, radiation-treated group (Fig.3B). Moreover, ki-67 expression was evaluated histologically. The findings showed that $A B Z$ and irradiation co-treatment induced significantly higher numbers of positive cells compared to vehicle (sesame oil), ABZ or X-ray irradiation treatment alone (Fig.3C). Furthermore, the number of TUNEL-positive cells was significantly higher in the ABZ and irradiation co-treatment groups than in vehicle (sesame oil), ABZ or X-ray irradiation treatment alone (Fig. 3C). bFGF promotes fiber collagen synthesis via stimulation of fibroblast differentiation, bFGF levels indirectly reflects the activity of basic fibroblast growth factor in tumor tissue. The Sirius Red Kit was used to stain the tissue to assess the amount of collagen fiber in tumor stroma. We demonstrated that the content of collagen fiber in ABZ- 
treated group was significantly higher than in control-treated group (Fig.3C). In summary, our results demonstrated that $A B Z$ enhanced the radiosensitivity of human pancreatic cancer cells to X-ray irradiation treatment via affecting.

\section{ABZ suppresses HIF-1a and bFGF expression in hypoxic pancreatic cancer cells in vitro and in vivo.}

We further explored the effect of ABZ on HIF-1a and bFGF expression in hypoxia pancreatic cancer cells in vitro and in PATU8988 xenograft in vivo. Decreased HIF-1 a and bFGF expression levels were observed in ABZ-treated hypoxic PC cells compared to those hypoxic PC cells without treatment (Fig.5A\&B). Furthermore, decreased HIF-1 $a$ and bFGF expression levels were observed in ABZ-treated mice compared to the vehicle-treated (sesame oil) mice (Fig.5C). We demonstrated that the content of collagen fiber in ABZ-treated group was significantly higher than in control-treated group (Fig.5C).

\section{Discussion}

Pancreatic cancer, the most malignant tumor among the gastrointestinal tumors, is known as a ' silent killer' due to its poor prognosis [21]. Advanced pancreatic cancer could be presented the time of diagnosis and occupy $80 \%$ of PC diseases, whereas radiotherapy is the mainstay treatment for PC [22]. Unfortunately, its therapeutic efficacy is limited by high tumor radioresistance and high-dose radiotherapy-induced side effect to patients. The tumor microenvironment (TME), especially that with high hypoxia is considered as the main cause of tumor radiotherapy resistance, and is closely related to hypoxia-inducible factor 1a \{Wachters, \#49;Green, 2007 \#50\}[23, 24]. In our study, we used cobalt chloride $\left(\mathrm{CoCl}_{2}\right)$ to mimic hypoxia condition and found that $\mathrm{CoCl}_{2}$ increased HIF-1a expression in a dosedependent manner. Moreover, the upregulation expression of HIF-1a was closely associated with the increased colony formation efficiency under radiation condition which is consistent with a previous study [25]. Futhermore, we found the expression of bFGF protein increases with increase in expression of hypoxia-induced hif-1a protein. These findings show that HIF-1a and bFGF activities are linked. On the one hand, Friedman et al and Houghton et al $[26,27]$, reported that hypoxia induces the secretion of bFGF and consequent enhancement of tumor resistance to irradiation. Further, studies have reported that bFGF contributes to tumor vessel dysfunction, hypoperfusion and increased tumor hypoxia by promoting the formation of cancer associated fibroblasts (CAFs) from fibroblasts [28]. CAFs may directly increase the radiotherapy resistance of tumors [29]. In addition, bFGF affects the secretion of HIF-1a-dependent VEGF closely related to radiation resistance [30].

$A B Z$, an anti-intestinal parasite drug, has been used in clinical treatment since 1987 [31]. Its safety has been approved in several clinical applications. Therefore, we used ABZ to explore its effect on tumor radiosensitivity and explore the underlying mechanisms. The effect of ABZ on HIF-1a and VEGF expression levels has been reported in in studies $[32,33]$. This study reports that ABZ could simultaneously inhibit HIF-1a and bFGF expression induced by hypoxia. This property increases the 
sensitivity of tumor cells to radiation in vitro and significantly inhibits human pancreatic cancer growth in vivo. These findings indicate that $A B Z$ is a potential radiotherapy sensitization agent.

In conclusion, we demonstrate that $A B Z$ enhances radiosensitivity of pancreatic cancer and its mechanism of action is by suppression of HIF-1a and bFGF expression. Our experimental data supports that $A B Z$ is a promising drug to improve the efficacy of radiation therapy for its antihypoxia effect.

\section{Conclusion}

In conclusion, we demonstrate that $A B Z$ enhances radiosensitivity of pancreatic cancer and its mechanism of action is by suppression of HIF-1a and bFGF expression. Our experimental data supports that $A B Z$ is a promising drug to improve the efficacy of radiation therapy for its antihypoxia effect.

\section{Declarations}

\section{Ethics approval and consent to participate}

All animal experiments were performed in strict accordance with the principles and procedures of Guide for the Care and Use of Laboratory Animal by the National Institutes of Health.

\section{Consent for publication}

Not applicable.

\section{Availability of data and materials}

The datasets used and/or analysed during the current study are available from the corresponging author on reasonable request.

\section{Abbreviations}

ABZ: Albendazole

PC: pancreatic cancer

HIF-1a: hypoxia-inducible factor 1-alpha

bFGF: basic fibroblast growth factor 
$\mathrm{CoCl}_{2}$ : Cobalt chloride

TME: tumor microenvironment

CAFs: cancer associated fibroblasts

\section{Competing interests}

There are no conflicts of interest.

\section{Funding}

This project was supported by the Key Research and Development Projects of Jiangsu Province 『No.BE2018659『and National Natural Science Foundation of China (81700129).

\section{Author information}

Haifeng Chen, Xiaochong Zhou, and Zhen Weng have contributed equally to this work and should be considered as joint first author.

\section{Affiliations}

Department of Gastroenterology, The First Affiliated Hospital of Soochow University, Suzhou,China;

Haifeng Chen, Xiaochong Zhou, Xing Wei,Chunfang Xu

Department of Hematological Disease Engineering Center Of Ministry Of Education, Soochow University, Suzhou, China.

Zhen Weng, Yang He

\section{Contributions}

Haifeng Chen designed the study. Haifeng Chen, Xiaochong Zhou and Xing Wei contributed to collecting the data, carring out data analyses and drafting the manuscript. This manuscript was revised by Zhen Weng. Chunfang Xu and Yang He conceived and supervised the entire project. All authors read and approved the final manuscript.

\section{Corresponding authors}


Correspondce to Chunfang Xu or Yang He.

\section{Acknowledgement}

Not applicable.

\section{References}

1. Macedo F, Cass S, Song N, Kelly K, Franceschi D, Yakoub D, Dudeja V, Livingstone A, Merchant N: Large cell and small cell pancreatic cancer: long-term outcomes from a nationwide cohort. $H P B$ 2019, 21:S121-S122.

2. Pan P, Yang BX, Ge XL: Brucea???javanica seed oil enhances the radiosensitivity of esophageal cancer by inhibiting hypoxia-inducible factor $1 \mathrm{a}$, in???vitro and in???vivo. Oncology Letters.

3. Yechieli RL, Robbins JR, Mahan M, Siddiqui F, Ajlouni M: Stereotactic Body Radiotherapy for Elderly Patients With Medically Inoperable Pancreatic Cancer. Am J Clin Oncol 2014, 87:22-26.

4. Luo F: Predictive modeling for in-hospital mortality in pancreatic cancer patients. Rutgers UniversitySchool of Health Professions, 2015.

5. Tan X, Chen J, Ren L, Lin R, Chen Z: Analgesic effect of high intensity focused ultrasound in patients with advanced pancreatic cancer. The Chinese-German Journal of Clinical Oncology 2013, 12:385388.

6. Yamashina T, Takada R, Uedo N, Akasaka T, Hanaoka N, Takeuchi Y, Higashino K, loka T, Ishihara R, Teshima T: Prospective small bowel mucosal assessment immediately after chemoradiotherapy of unresectable locally advanced pancreatic cancer using capsule endoscopy: a case series. Annals of Gastroenterology: Quarterly Publication of the Hellenic Society of Gastroenterology 2016, 29:386.

7. Myrehaug S, Sahgal A, Russo SM, Lo SS, Rosati LM, Mayr NA, Lock M, Small W, Dorth JA, Ellis RJ: Stereotactic body radiotherapy for pancreatic cancer: recent progress and future directions. Expert Review of Anticancer Therapy:14737140.14732016.11168698.

8. Liu Y, Zhang Z, Wang J, Chen C, Tang X, Zhu J, Liu J: Metabolic reprogramming results in abnormal glycolysis in gastric cancer: a review. OncoTargets and therapy 2019, 12:1195.

9. Michaylira CZ, Nakagawa H: Hypoxic microenvironment as a cradle for melanoma development and progression. Cancer biology \& therapy 2006, 5:476-479.

10. Hesari A, Rezaei M, Rezaei M, Dashtiahangar M, Fathi M, Rad JG, Momeni F, Avan A, Ghasemi F: Effect of curcumin on glioblastoma cells. Journal of cellular physiology 2019, 234:10281-10288.

11. Debeb BG, Xu W, Woodward WA: Radiation resistance of breast cancer stem cells: understanding the clinical framework. Journal of mammary gland biology and neoplasia 2009, 14:11-17. 
12. De Wever O, Mareel M: Role of tissue stroma in cancer cell invasion. The Journal of Pathology: A Journal of the Pathological Society of Great Britain and Ireland 2003, 200:429-447.

13. Fan R, Hou W-J, Zhao Y-J, Liu S-L, Qiu X-S, Wang E-H, Wu G-P: Overexpression of HPV16 E6/E7 mediated HIF-1a upregulation of GLUT1 expression in lung cancer cells. Tumor Biology, 37:46554663.

14. Evrard B, Chiap P, Detullio P, Ghalmi F, Delattre L: Oral bioavailabilty in sheep of albendazole from a suspension and from a solution containing hydroxypropyl-??-cycldextrin. Journal of Controlled Release 2002, 85:45-50.

15. Pourgholami MH, Cai ZY, Chu SW, Galettis P, Morris DL: The influence of ovarian cancer induced peritoneal carcinomatosis on the pharmacokinetics of albendazole in nude mice. Anticancer research 2010, 30:423-428.

16. Zhou F, Du J, Wang J: Albendazole inhibits HIF-1a-dependent glycolysis and VEGF expression in nonsmall cell lung cancer cells. Molecular and cellular biochemistry 2017, 428:171-178.

17. Subramanian VS, Subramanya SB, Said HM: Relative contribution of THTR-1 and THTR-2 in thiamin uptake by pancreatic acinar cells: studies utilizing Slc19a2 and Slc19a3 knockout mouse models. Ajp Gastrointestinal \& Liver Physiology, 302:G572-G578.

18. Hardin T, Najvar L, Rizzo J, Fothergill A, Rinaldi M, Graybill J: Discrepancy between in vitro and in vivo antifungal activity of albendazole. Journal of medical and veterinary mycology 1997, 35:153-158.

19. Krajewski S, Krajewska M, Shabaik A, Wang H-G, Irie S, Fong L, Reed JC: Immunohistochemical analysis of in vivo patterns of Bcl-X expression. Cancer Research 1994, 54:5501-5507.

20. Lund DD, Twietmeyer TA, Schmid PG, Tomanek RJ: Independent changes in cardiac muscle fibres and connective tissue in rats with spontaneous hypertension, aortic constriction and hypoxia. 1979, 13:39-44.

21. He Y, Zheng R, Li D, Zeng H, Zhang S, Chen W: Pancreatic cancer incidence and mortality patterns in China, 2011. Chinese Journal of Cancer Research 2015, 27:29.

22. DPC4 Gene Status of the Primary Carcinoma Correlates With Patterns of Failure in Patients With Pancreatic Cancer. Journal of Clinical Oncology, 27:1806-1813.

23. Green MM, Hiley CT, Shanks JH, Bottomley IC, West CM, Cowan RA, Stratford IJ: Expression of vascular endothelial growth factor (VEGF) in locally invasive prostate cancer is prognostic for radiotherapy outcome. International Journal of Radiation Oncology* Biology* Physics 2007, 67:8490.

24. Hsieh C-H, Wu C-P, Lee H-T, Liang J-A, Yu C-Y, Lin Y-J: NADPH oxidase subunit 4 mediates cycling hypoxia-promoted radiation resistance in glioblastoma multiforme. Free Radical Biology \& Medicine, 53:649--658.

25. Zhou F, Du J, Wang J: Albendazole inhibits HIF-1a-dependent glycolysis and VEGF expression in nonsmall cell lung cancer cells. Molecular and Cellular Biochemistry, 428:171-178.

26. Haimovitz-Friedman A, Balaban NA, Mcloughlin M, Ehleiter D, Fuks Z: Protein kinase C mediates basic fibroblast growth factor protection of endothelial cells against radiation-induced apoptosis. 
Cancer Research 1994, 54:2591-2597.

27. Li H, Fan X, Houghton J: Tumor microenvironment: The role of the tumor stroma in cancer. Journal of Cellular Biochemistry 2007, 101:805-815.

28. Hao J, Ding X-L, Yang X, Wu X-Z: Prunella vulgaris polysaccharide inhibits growth and migration of breast carcinoma-associated fibroblasts by suppressing expression of basic fibroblast growth factor. Chinese Journal of Integrative Medicine 2016:1-7.

29. Majidinia M, Yousefi B: Breast tumor stroma: A driving force in the development of resistance to therapies. Chemical Biology \& Drug Design, 89:309-318.

30. Shi YH, Wang YX, Bingle L, Gong LH, Heng WJ, Li Y, Fang WG: In vitro study of HIF-1 activation and VEGF release by bFGF in the T47D breast cancer cell line under normoxic conditions: involvement of PI-3K/Akt and MEK1/ERK pathways. Journal of Pathology 2005, 205:530-536.

31. Lanchote V, Takayanagui $\mathrm{O}$, Mateus F: Enantioselective renal excretion of albendazole metabolites in patients with neurocysticercosis. Chirality: The Pharmacological, Biological, and Chemical Consequences of Molecular Asymmetry 2004, 16:520-525.

32. Pourgholami MH, Cai ZY, Badar S, Wangoo K, Poruchynsky MS, Morris DL: Potent inhibition of tumoral hypoxia-inducible factor $1 \mathrm{a}$ by albendazole. 10:143-140.

33. Veronika H, Lenka S, Vera K, Petra M: Potential Anti-cancer Drugs Commonly Used for Other Indications. Current Cancer Drug Targets, 15:35-52.

\section{Figures}


A

PATU8988

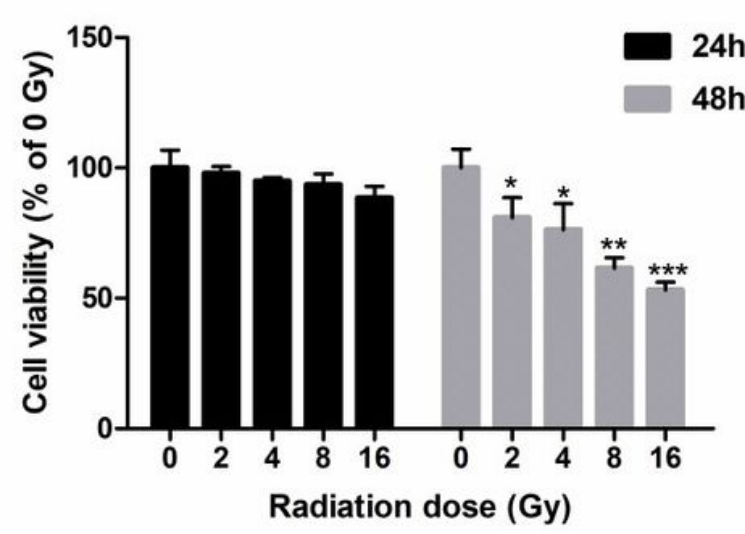

C
B

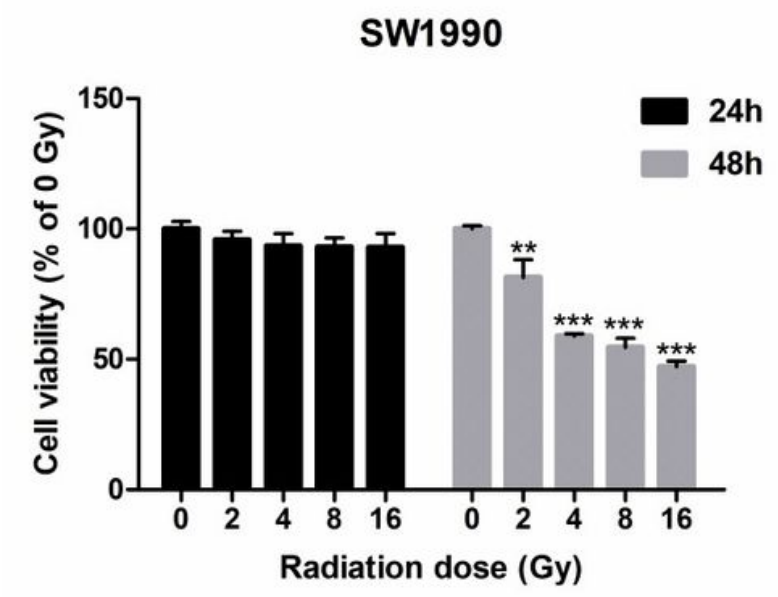

D
PATU8988

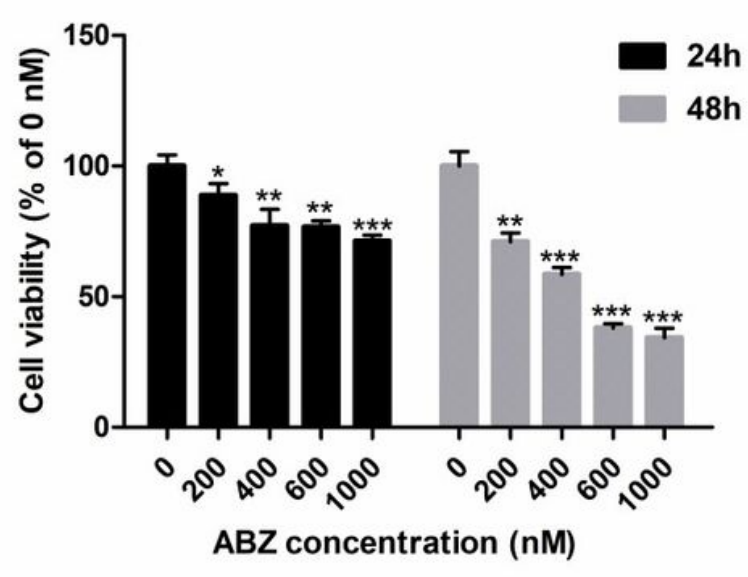

SW1990

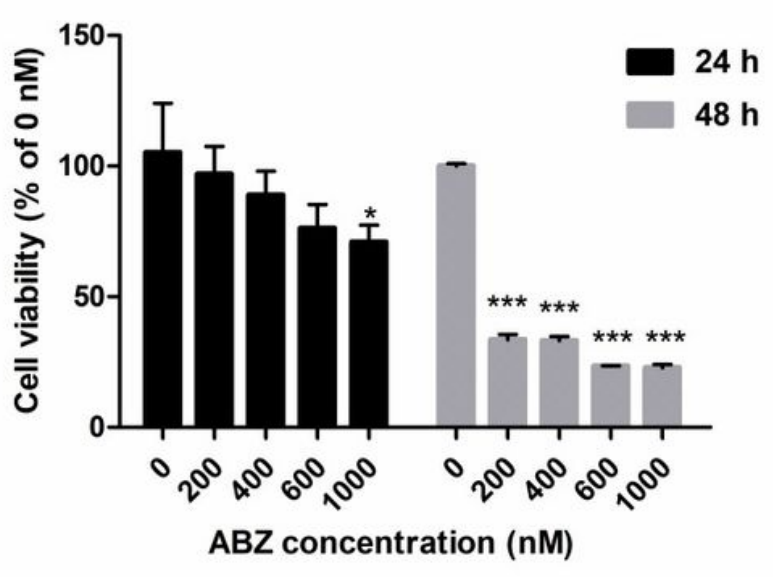

Figure 2

Radiation dosage and ABZ treatment concentration determination in pancreatic cancer cell lines using MTT cell viability analysis. A. PATU8988 cells treated with different dosages of radiation, B. SW1990 cells treated with different dosages of radiation, C. PATU8988 cells treated with different dosages of ABZ, D. SW1990 cells treated with different dosages of radiation. The results showed that $4 \mathrm{~Gy}$ radiation dosage and $200 \mathrm{nM}$ ABZ could result in significant effects on cell viability. ${ }^{*} P<0.05 \rrbracket * * P<0.01$ and ${ }^{* \star *} P<0.001$ compared to radiation dosage at $0 \mathrm{~Gy}$ and $A B Z$ at $0 \mathrm{~nm}$ at the same time point. 
A

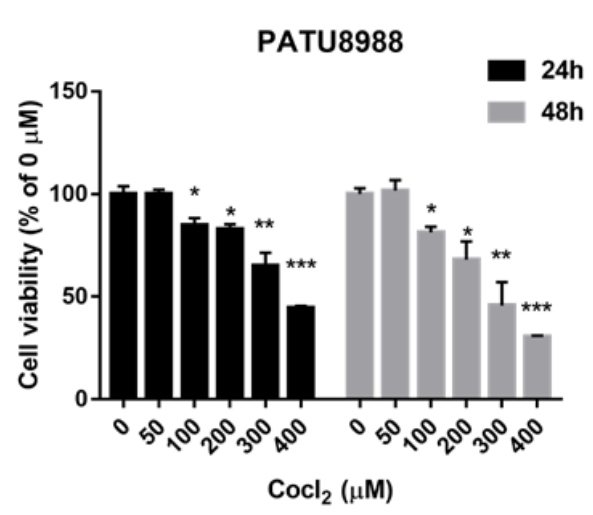

C

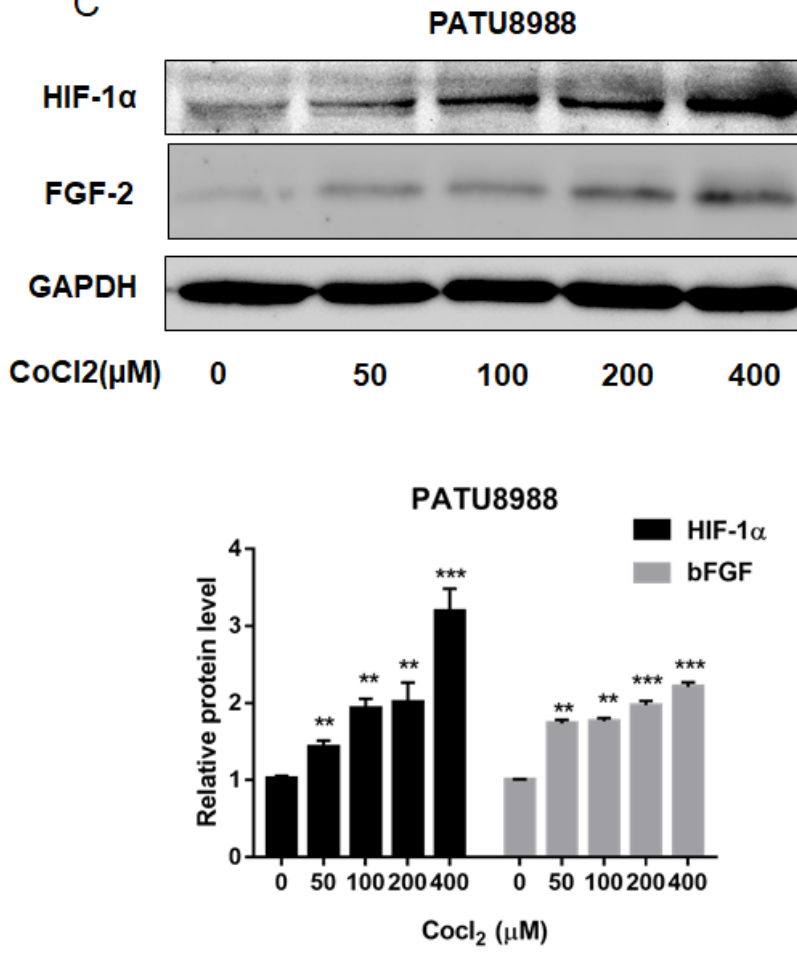

B
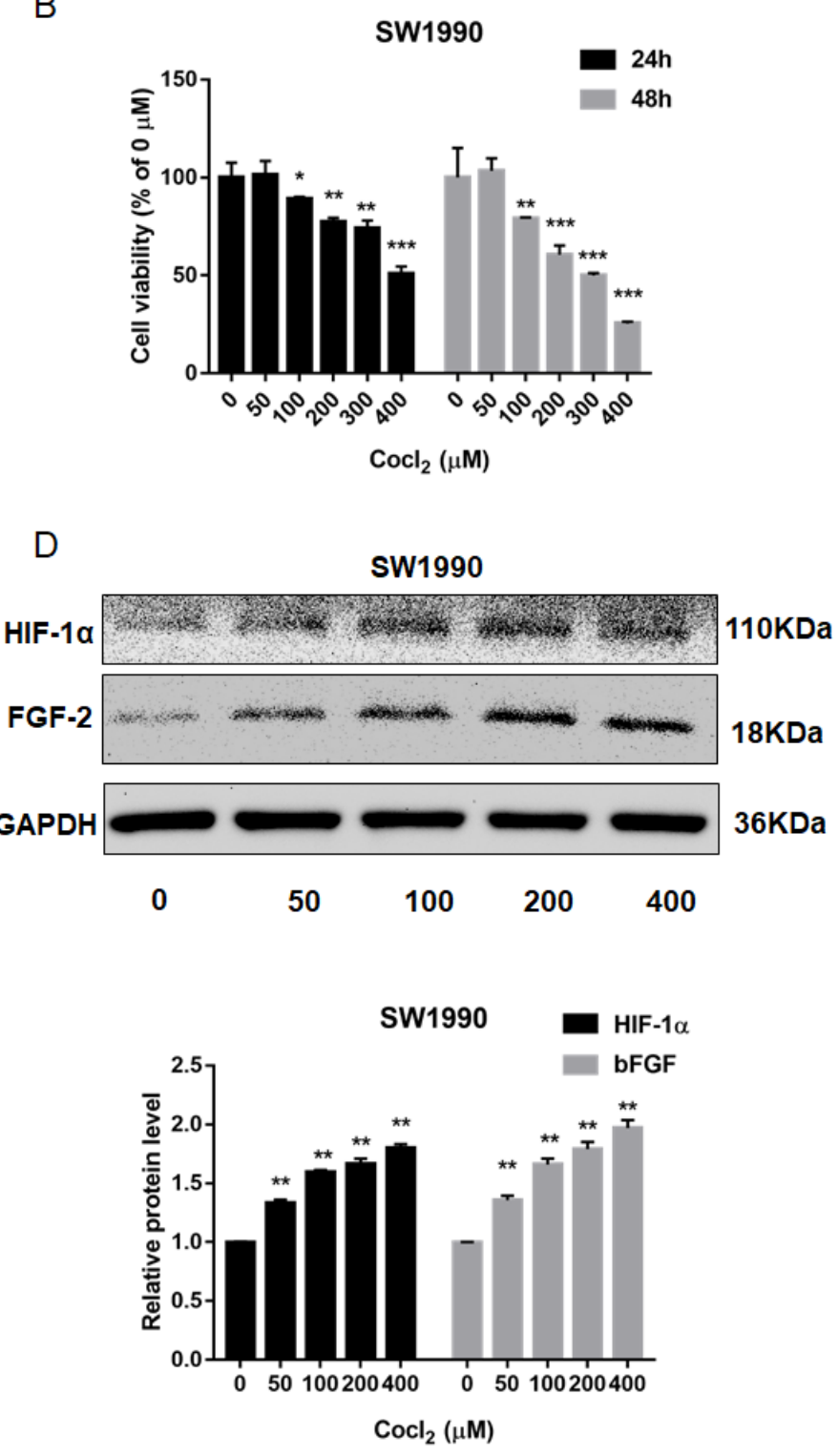

Figure 4

Hypoxia induction condition optimization via pancreatic cancer cell lines treated with series concentration of CoCl2. Cell viability of PATU8988 (A) and SW1990 (B) after series concentration of $\mathrm{CoCl} 2$ incubation. Effects of $\mathrm{CoCl} 2$ induced hypoxia condition on HIF-1a and FGF-2 in PATU8988 (C) and SW1990 (D) after series concentration of $\mathrm{CoCl} 2$ incubation. ${ }^{*} \mathrm{P}<0.05, * * P<0.01, * \star * P<0.001$ compared to $\mathrm{CoCl} 2$ concentration at $0 \mu \mathrm{M}$, whereas ${ }^{* *} \mathrm{p}<0.01$ and ${ }^{* \star *} \mathrm{p}<0.001$ for corresponding protein level comparison when $\mathrm{CoCl} 2$ concentration at $0 \mu \mathrm{M}$. 
A

PATU8988

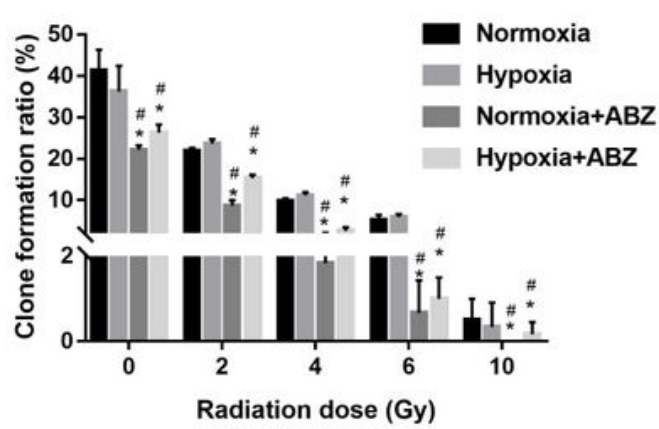

B

sW1990

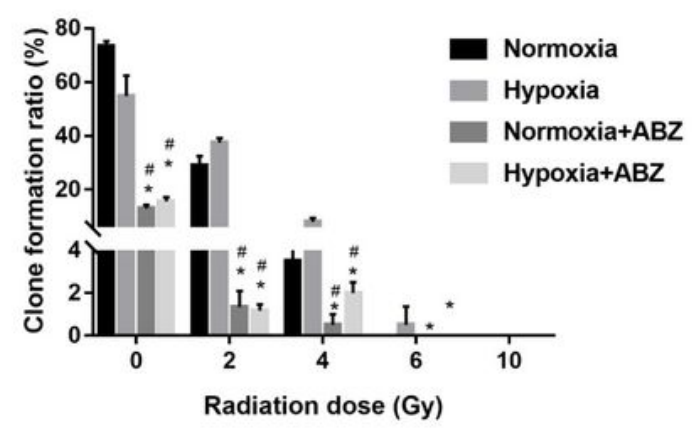

C

Radiation
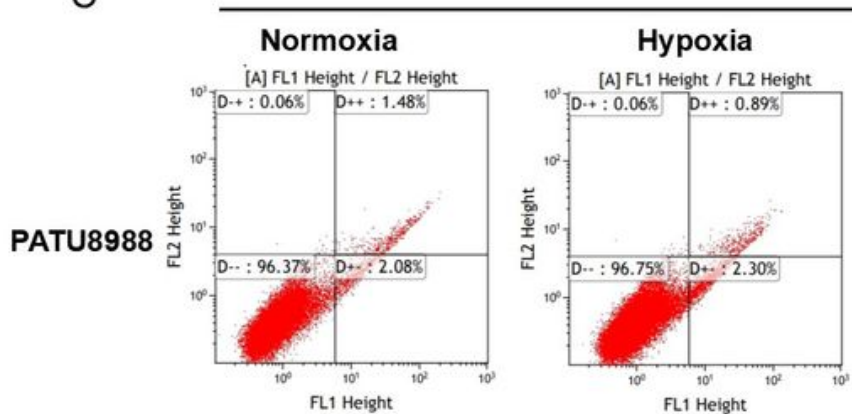

Normoxia +ABZ
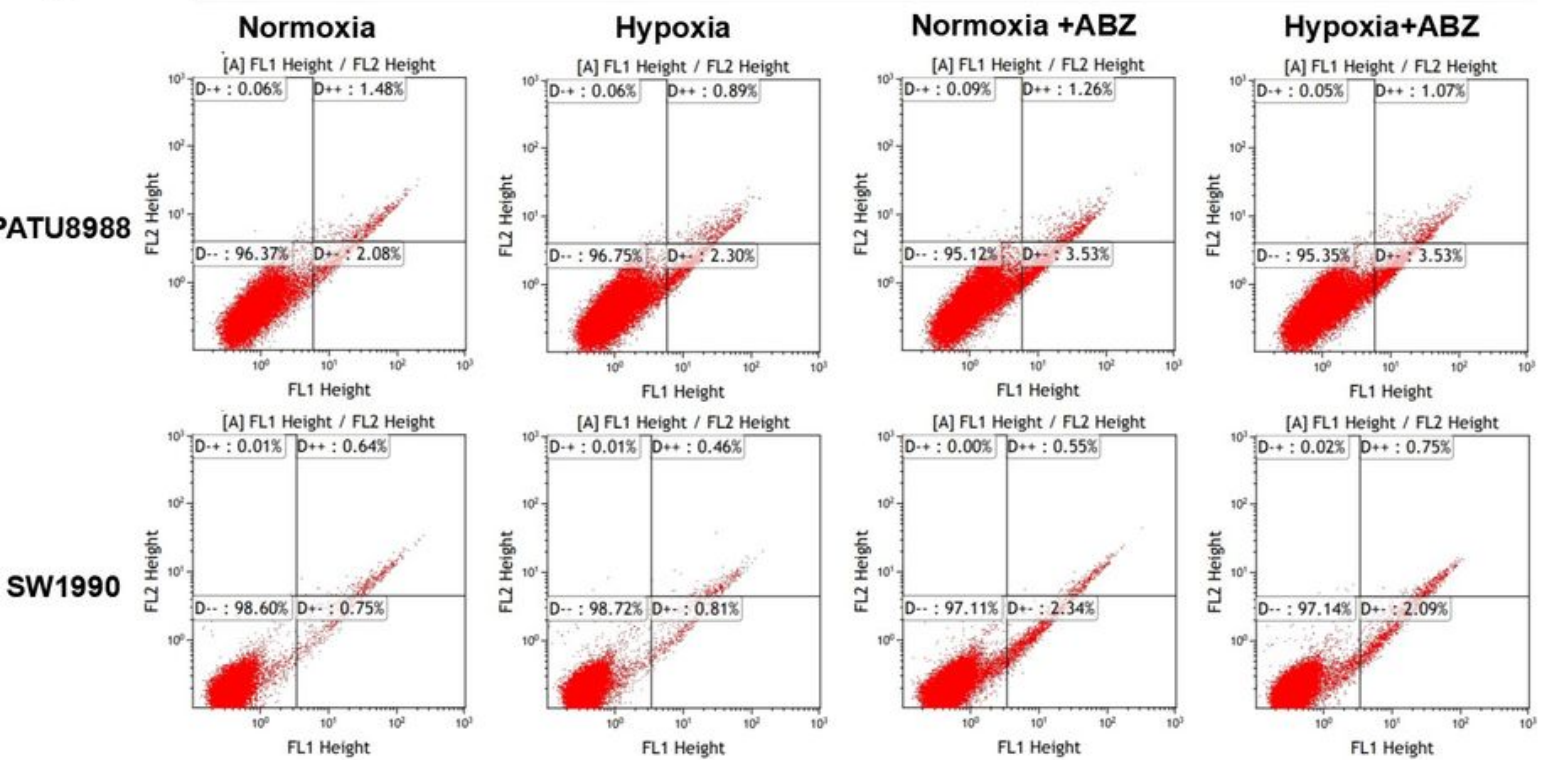

PATU8988

\section{SW1990}
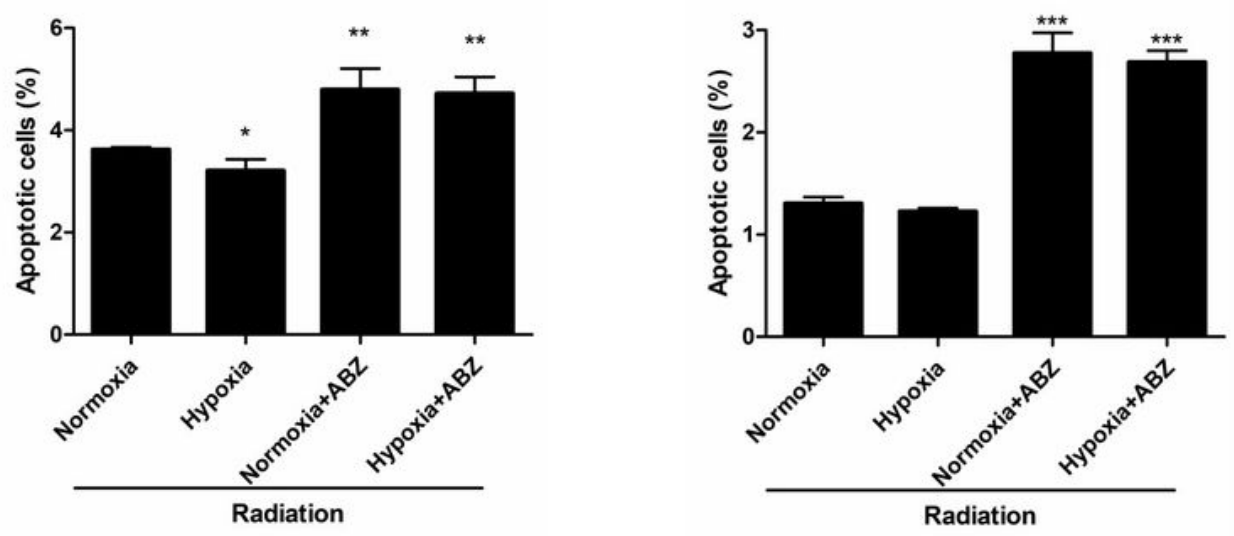

\section{Figure 6}

$A B Z$ enhanced the sensitivity of pancreatic cancer cell lines to radiation illustrated by decreased clone formation and increased cell apoptosis. A. Clone formation of PATU8988, B. Clone formation of SW1990, C. Cell apoptosis of PATU8988 and SW1990. ${ }^{*} p<0.05$ and ${ }^{*} * \mathrm{k}<0.001$ compared to cell under normoxia condition, \#p<0.05 compared to cells under hypoxia condition. 
A

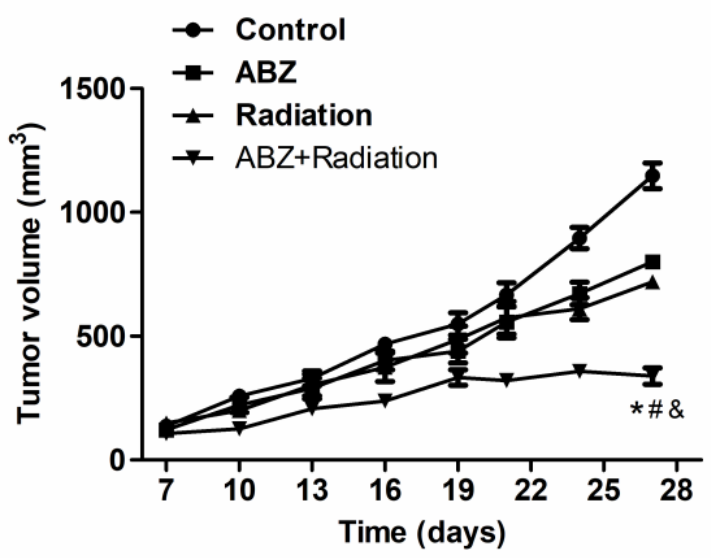

C
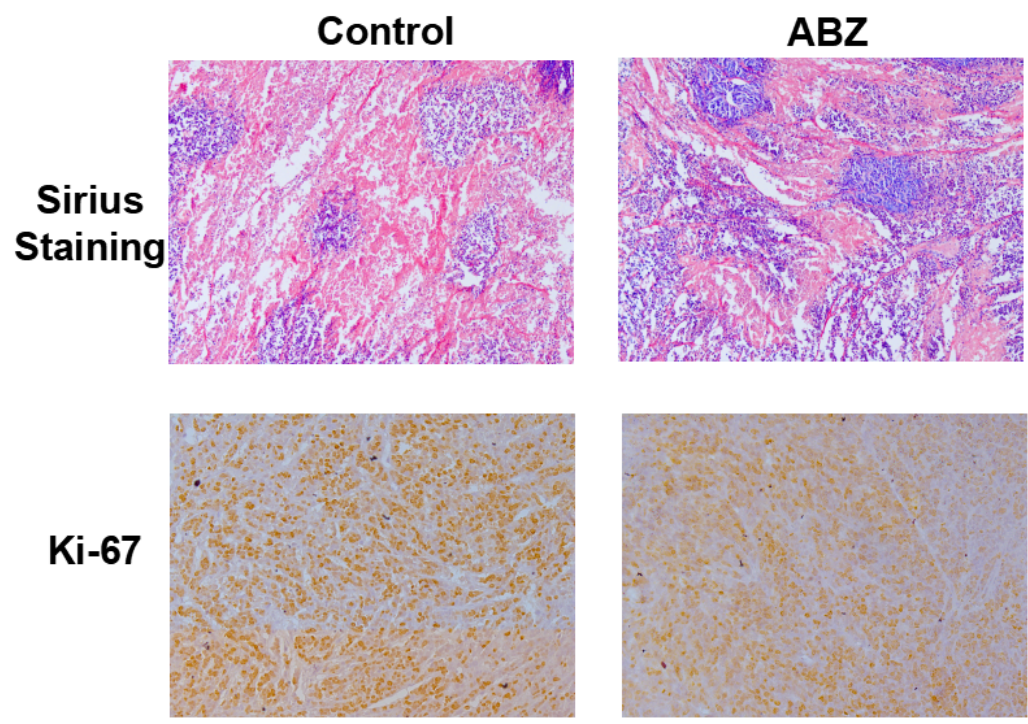

Ki-67

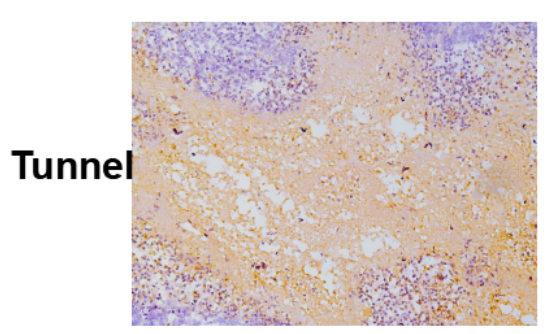

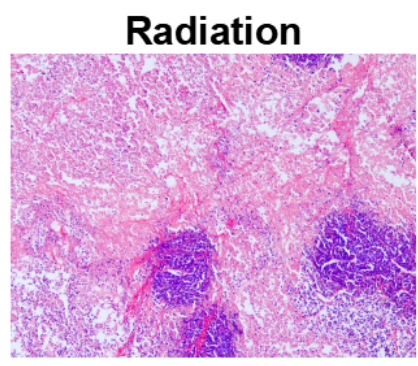

B
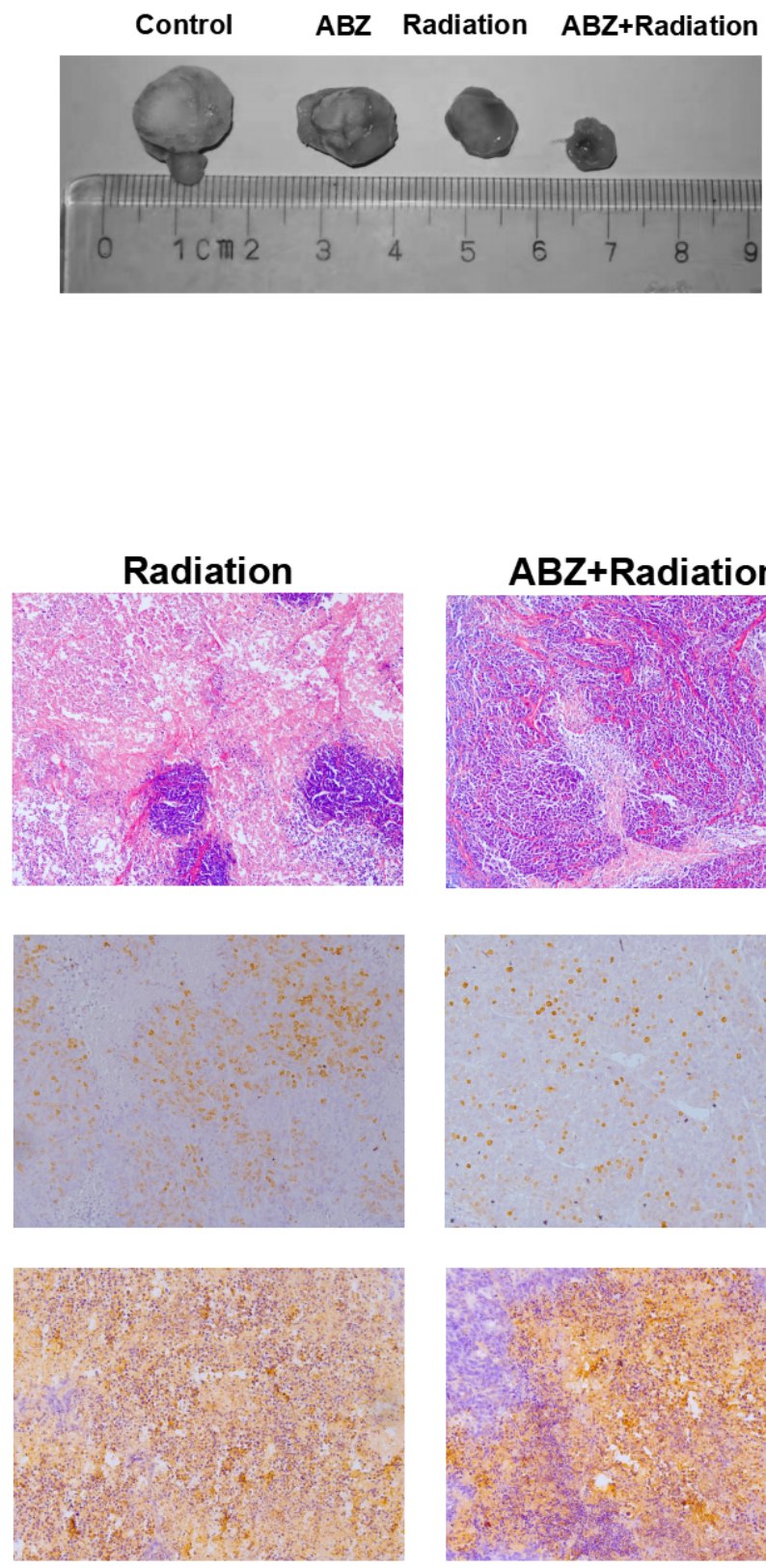
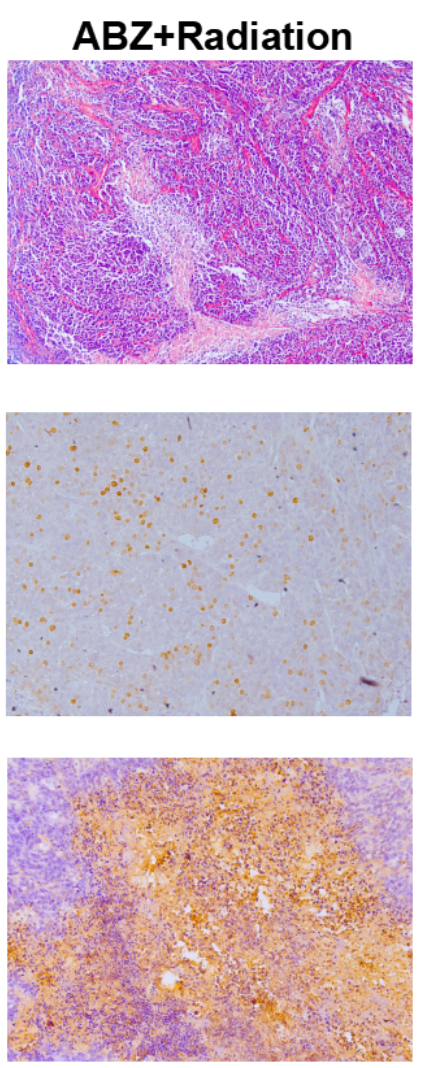

\section{Figure 8}

ABZ significantly enhanced the pancreatic cancer growth inhibition effects by radiation in vivo. An ectopic xenograft tumor in vivo model was established, followed by tumor volume measurement and immunohistochemistry evaluation. A. Tumor volume measurement at different time points in different group of treatment, and significantly decreased tumor volume could be found in $A B Z+$ Radiation treatment compared to $A B Z$ and radiation alone treatment. B. Representative images of the tumor with different treatment. C. ABZ and radiation combined treatment could result in significantly decreased collagen deposit illustrated by Sirius staining, decreased cell proliferation illustrated by Ki-67, increased 
cell apoptosis indicated by TUNEL staining compared to ABZ and radiation alone. * $\mathrm{p}<0.05$ compared to control group, \#p<0.05 compared to $A B Z$ group, \&p<0.05 compared to radiation group.

A

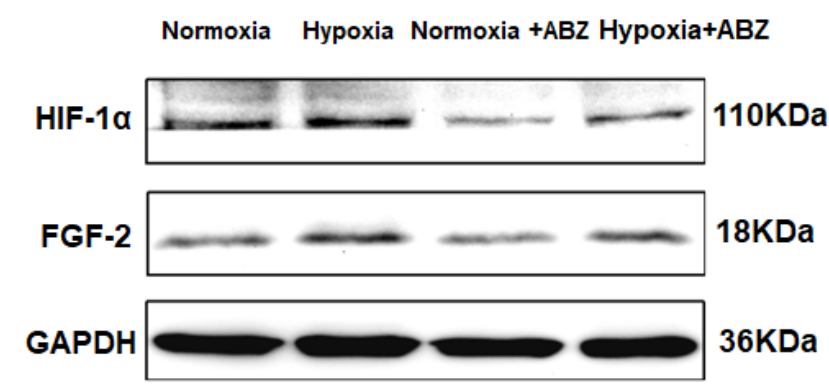

PATU8988

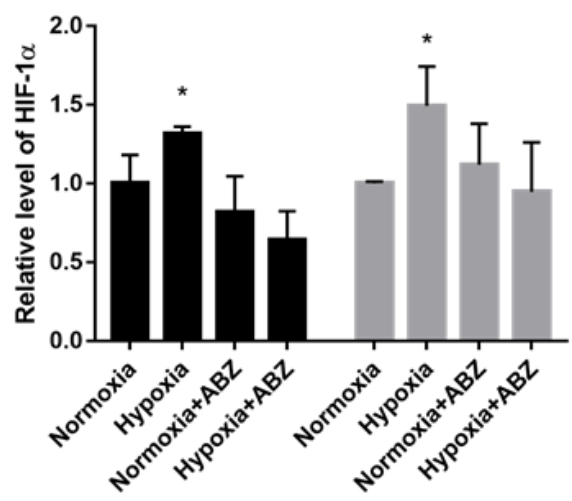

C Control ABZ Radiation ABZ+Radiation

HIF-10

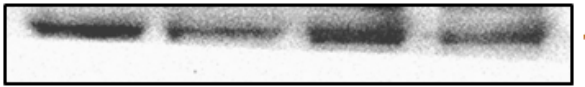

$110 \mathrm{KDa}$

FGF-2

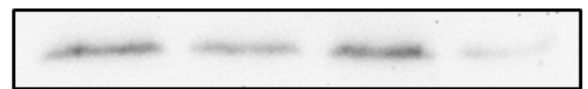

$18 \mathrm{KDa}$

GAPDH

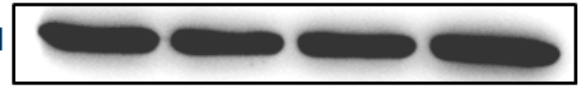

$36 \mathrm{KDa}$
B
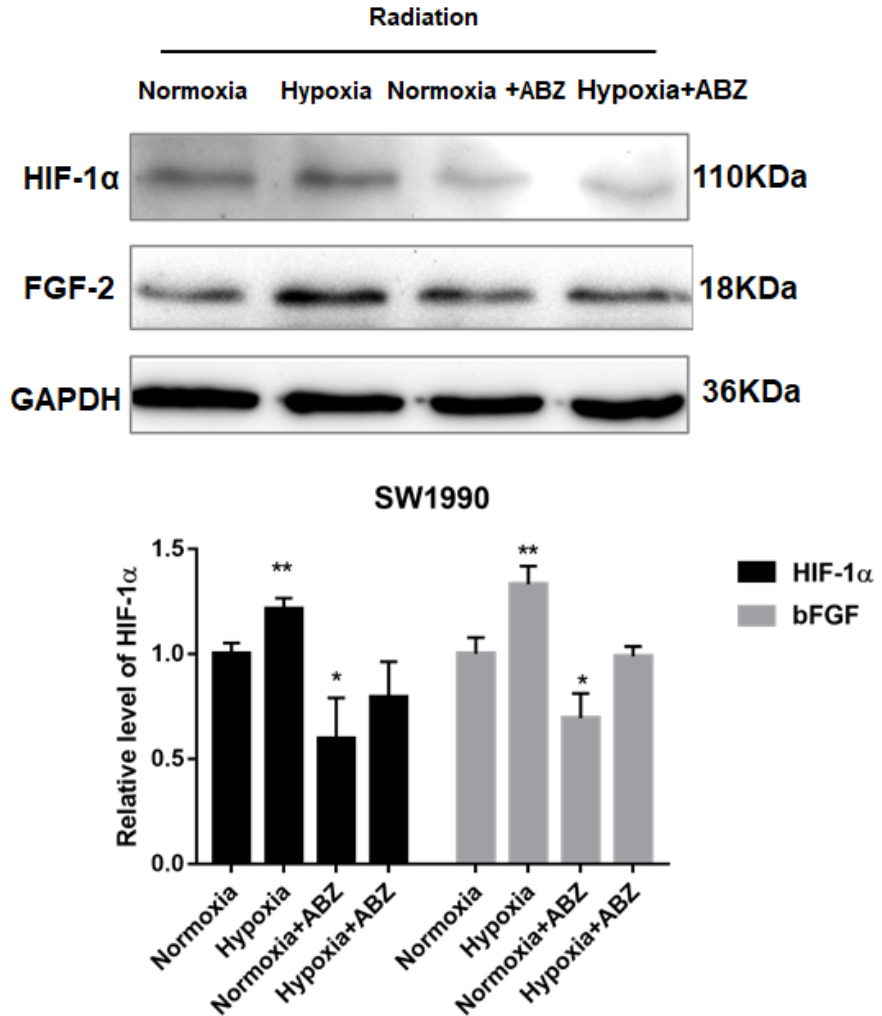

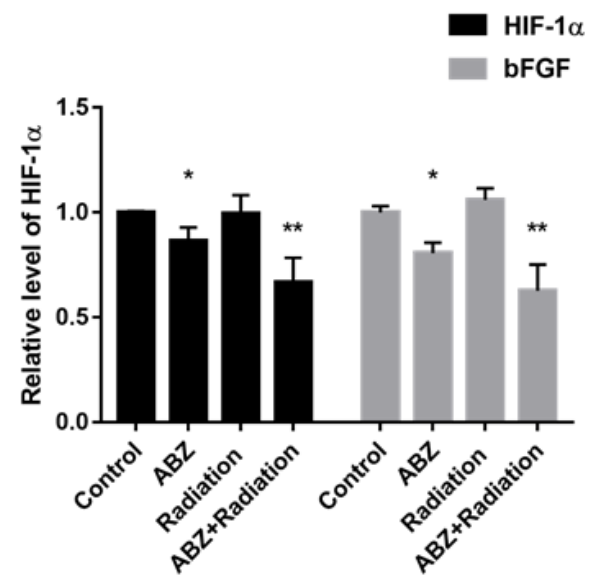

Figure 10

ABZ suppresses HIF-1 $a$ and bFGF expression in hypoxic pancreatic cancer cells in vitro and in vivo. A. Decreased HIF-1a and bFGF expression levels were observed in ABZ-treated hypoxic PC cells PATU8988 compared to those hypoxic PC cells without treatment, B. Decreased HIF-1a and bFGF expression levels were observed in ABZ-treated hypoxic PC cells SW1990 compared to those hypoxic PC cells without treatment, C. Decreased HIF-1a and bFGF expression levels were observed in ABZ-treated mice compared to the vehicle-treated (sesame oil) mice. ${ }^{*} p<0.05$ and ${ }^{* *} p<0.01$ compared to normoxia group in vitro or control group in vivo. 